\title{
Diffusioosmosis of electrolyte solutions in a capillary slit with adsorbed polyelectrolyte layers
}

\author{
Hsien Chen Ma, Huan J. Keh* \\ Department of Chemical Engineering, National Taiwan University, Taipei 10617, Taiwan, ROC
}

Received 23 March 2007; accepted 3 May 2007

Available online 13 June 2007

\begin{abstract}
A theoretical study is presented for the steady diffusioosmotic flow of an electrolyte solution in a fine capillary slit with each of its inside walls coated with a layer of polyelectrolytes generated by an imposed tangential concentration gradient. In this solvent-permeable and ion-penetrable surface charge layer, idealized polyelectrolyte segments are assumed to be distributed at a uniform density. The electric double layer and the surface charge layer may have arbitrary thicknesses relative to the gap width between the slit walls. The Poisson-Boltzmann equation and a modified Navier-Stokes/Brinkman equation are solved numerically to obtain the electrostatic potential, dynamic pressure, tangentially induced electric field, and fluid velocity as functions of the lateral position in the slit in a self-consistent way, with the constraint of no net electric current arising from the cocurrent diffusion, electric migration, and diffusioosmotic convection of the electrolyte ions. The existence of the surface charge layers can lead to a diffusioosmotic flow quite different from that in a capillary with bare walls. The effect of the lateral distribution of the induced tangential electric field and the relaxation effect due to ionic convection in the slit on the diffusioosmotic flow are found to be very significant in practical situations.
\end{abstract}

(C) 2007 Elsevier Inc. All rights reserved.

Keywords: Diffusioosmosis; Polyelectrolyte-coated capillary; Electrokinetic flow; Arbitrary double-layer thickness; Relaxation effect

\section{Introduction}

The electrokinetic flows of an electrolyte solution in a small pore with a charged wall are of much fundamental and practical interest in various areas of science and engineering. Perhaps the most familiar example of an electrokinetic flow is electroosmosis, which results from the interaction between a tangentially applied electric field and the electric double layer adjacent to the charged wall. Problems of fluid flow caused by this wellknown mechanism were studied extensively in the past [1-10].

Another example of electrokinetic flows in a micropore, which is termed diffusioosmosis (also known as capillary osmosis $[5,11])$ and has caught less attention, involves a tangential concentration gradient of the electrolyte that interacts with the charged pore wall. As in the case of electroosmosis, the electrolyte-wall interaction in diffusioosmosis is electrostatic

\footnotetext{
* Corresponding author.

E-mail address: huan@ntu.edu.tw (H.J. Keh).
}

in nature and its range is the Debye screening length $\kappa^{-1}$ (defined right after Eq. (3)). The tangential gradient of a dissociating electrolyte produces fluid flow along a charged solid surface by two mechanisms. The first comprises the stresses developed by the tangential gradient of the excess pressure within the electric double layer (chemiosmotic effect), and the second is based on the macroscopic electric field that is generated because the tangential diffusive and convective fluxes of the two electrolyte ions are not equal (electroosmotic effect). The fluid motion caused by diffusioosmosis has been analytically examined for electrolyte solutions near a plane wall [11-17] and inside a capillary pore [17-23]. Some experimental results and interesting applications concerning diffusioosmosis are also available in the literature [24].

Although the basic relationships involved in electrokinetic phenomena were derived mainly by using the traditional model of plane distribution of surface charges, quite a number of investigations have applied these phenomena to the study of the effects of adsorbed polyelectrolytes on the solid surfaces. The electroosmotic flows in capillaries with thin polymer layers on 
the inside walls were theoretically examined for the cases of a slit $[25,26]$ and a tube [27] with thin double layers. On the other hand, analytical formulas for the electroosmotic velocity profile of electrolyte solutions on the cross section of a capillary with its inside wall coated with a finite layer of adsorbed polyelectrolytes were obtained by solving the linearized Poisson-Boltzmann equation for the case of an arbitrary value of $\kappa R$ or $\kappa h$, where $R$ is the radius of a capillary tube and $h$ is the half-thickness of a capillary slit $[28,29]$.

Recently, the diffusioosmotic flow of a solution of symmetric electrolytes in a fine capillary slit bearing adsorbed polyelectrolytes on its inside walls was theoretically investigated for the case of small electrostatic potentials or low fixed-charge densities, and analytical formulas for the fluid velocity profile on the cross section of the slit were obtained [30,31]. In these studies, however, either the effect of lateral distributions of the counterions and co-ions (or of the electrostatic potential) on the local electric field induced by the imposed electrolyte concentration gradient in the tangential direction or the effect of the ionic convection on it caused by the diffusioosmotic flow (relaxation effect) was neglected. Moreover, these analyses are subject to the severe restriction that the electrostatic potential is sufficiently low (less than about $25 \mathrm{mV}$ ) for the Debye-Hückel approximation to be acceptable. In practical applications, however, electrostatic potentials as high as $100-200 \mathrm{mV}$ are frequently encountered.

In this work we present a comprehensive analysis of the steady diffusioosmosis of an electrolyte solution with a constant prescribed concentration gradient through a capillary slit bearing permanently adsorbed or covalently bound polyelectrolytes on each of its inside walls. The charge and segment densities of the adsorbed polymers are assumed to be uniform throughout the surface charge layer, but no assumption is made concerning the magnitude of the electrostatic potential or the thicknesses of the surface charge layer and the electric double layer relative to the gap width between the slit walls. Both the lateral distribution of the induced axial electric field and the effect of the ionic convection on it are allowed. The PoissonBoltzmann equation governing the electrostatic potential and a modified Navier-Stokes/Brinkman equation governing the fluid flow within the capillary are numerically solved and results for the induced electric field and diffusioosmotic velocity profiles are obtained for various cases. These results show that the effect of the deviation of the induced electric field in the slit from its bulk-phase quantity and the effect of the ionic convection on the diffusioosmotic velocity of the fluid are very significant in practical situations.

\section{Electrostatic potential distribution}

In this section we consider the lateral distribution of the electrostatic potential in the fluid solution of a symmetrically charged electrolyte of valence $Z$ (where $Z$ is a positive integer) undergoing diffusioosmosis in the narrow channel between two large identical parallel plates of length $L$ at separation distance $2 h$ with $h \ll L$, as illustrated in Fig. 1 . Each of the inside walls of the capillary slit is coated with a layer of adsorbed

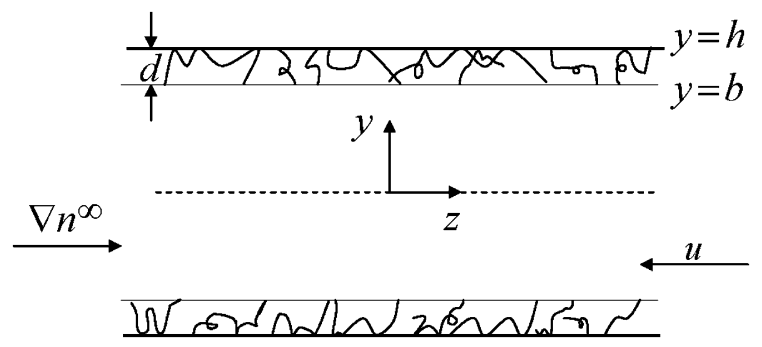

Fig. 1. Geometrical sketch for diffusioosmosis in a capillary slit with each of its inside walls coated with a layer of adsorbed polyelectrolytes.

charged polymers in equilibrium with the surrounding solution. The polymer layer is treated as a solvent-permeable and ionpenetrable surface charge layer of constant thickness $d=h-b$ in which fixed-charged groups of valence $q$ are distributed at a uniform density $N$. Experimental values for human erythrocytes [32], rat lymphocytes [33], and grafted polymer macrocapsules [34] indicate that $d$ ranges from $7.8 \mathrm{~nm}$ to $3.38 \mu \mathrm{m}$ and $N$ can be as high as $0.03 \mathrm{kmol} / \mathrm{m}^{3}$, depending on the $\mathrm{pH}$ and ionic strength of the electrolyte solution.

The applied electrolyte concentration gradient $\nabla n^{\infty}$ is a constant along the axial ( $z$ ) direction in the capillary slit, where $n^{\infty}(z)$ is the linear concentration (number density) distribution of the electrolyte in the bulk solution phase in equilibrium with the fluid inside the slit. The electrolyte ions can diffuse freely in the slit (inside and outside the surface charge layers), so there exists no regular osmotic flow of the solvent. The end effects are neglected. It is assumed that $n^{\infty}$ is only slightly nonuniform, such that

$L\left|\nabla n^{\infty}\right| / n^{\infty}(0) \ll 1$,

where $z=0$ is set at the midpoint through the slit. Thus, the variation of the electrostatic potential (excluding the macroscopic electric field induced by the electrolyte gradient, which will be discussed in the next section) and ionic concentrations in the capillary with the axial position can be neglected in comparison with their corresponding quantities at $z=0$.

Owing to the planar symmetry of the system, we need consider only the half-region $0 \leqslant y \leqslant h$, where $y$ is the distance from the median plane between the slit walls in a normal direction. If $\psi(y)$ represents the electrostatic potential at the position $y$ relative to that in the bulk solution and $n_{+}(y, z)$ and $n_{-}(y, z)$ denote the local concentrations of the cations and anions, respectively, of the symmetric electrolyte with valence $Z$, then Poisson's equation gives

$\frac{\mathrm{d}^{2} \psi}{\mathrm{d} y^{2}}=-\frac{e}{\varepsilon}\left\{Z\left[n_{+}(y, 0)-n_{-}(y, 0)\right]+H(y) q N\right\}$.

In this equation, $e$ is the charge of a proton; $H(y)$ is a unit step function, which equals unity if $b<y<h$, and zero if $0 \leqslant$ $y<b ; \varepsilon$ is the permittivity of the electrolyte solution which is assumed to be constant.

The local ionic concentrations $n_{+}$and $n_{-}$can also be related to the electrostatic potential $\psi$ by the Boltzmann equation,

$n_{ \pm}=n^{\infty} \exp (\mp \bar{\psi})$ 
where $\bar{\psi}=Z e \psi / k T$ ( $k$ is the Boltzmann constant and $T$ is the absolute temperature) is the dimensionless potential profile. Substitution of Eq. (2) into Eq. (1) results in the well-known Poisson-Boltzmann equation,

$\frac{\mathrm{d}^{2} \bar{\psi}}{\mathrm{d} y^{2}}=\kappa^{2} \sinh \bar{\psi}-H(y) \kappa^{2} \bar{N}$,

where

$\kappa=\left[2(Z e)^{2} n^{\infty}(0) / \varepsilon k T\right]^{1 / 2}$

is the Debye screening parameter and

$\bar{N}=Z e^{2} q N / \varepsilon \kappa^{2} k T$.

Note that the Debye-Hückel approximation with small magnitudes of $\bar{\psi}$, which was employed in the previous studies, is not used here. Also, the parameter $\bar{N}$ is of order unity and can be viewed as a nondimensionalized Donnan potential [26,35] of the surface charge layer.

For the case of constant surface potential, the boundary conditions for $\bar{\psi}$ are

$\frac{\mathrm{d} \bar{\psi}}{\mathrm{d} y}(y=0)=0$,

$\bar{\psi}\left(y=b^{-}\right)=\bar{\psi}\left(y=b^{+}\right)$,

$\frac{\mathrm{d} \bar{\psi}}{\mathrm{d} y}\left(y=b^{-}\right)=\frac{\mathrm{d} \bar{\psi}}{\mathrm{d} y}\left(y=b^{+}\right)$,

$\bar{\psi}(y=h)=\bar{\zeta}$,

where $\bar{\zeta}=Z e \zeta / k T$ is the dimensionless zeta potential at the shear plane of the slit walls adjacent to the electrolyte solution having a uniform bulk concentration $n^{\infty}(0)$. Equations (4b) and $(4 \mathrm{c})$ are the continuity requirements for $\psi$ and $\mathrm{d} \psi / \mathrm{d} y$ at the outer edge of the surface charge layer $[26,36]$. If the constant surface charge density, instead of the surface potential $\zeta$, is known at the slit walls, the boundary condition specified by Eq. (4d) should be replaced by the Gauss condition [22].

Equations (3) and (4), with arbitrary values of $b / h, \bar{N}, \bar{\zeta}$, and $\kappa h$, can be numerically solved by employing the finite difference method. Figs. $2 \mathrm{a}$ and $2 \mathrm{~b}$ show the numerical results of the dimensionless potential $\bar{\psi}$ for the case of a slit filled with adsorbed polyelectrolytes $(b / h=0)$ and for the case of a slit with finite surface charge layers $(b / h=0.8)$, respectively, with $\bar{N}=1$ as functions of the relative position $y / h$ for several values of the parameters $\bar{\zeta}$ and $\kappa h$. As expected, the potential profile for any case of given values of $b / h$ and $\kappa h$ increases monotonically with an increase in $\bar{\zeta}$. The decay of the potential from the capillary wall with decreasing $y / h$ becomes gentler when the value of $\kappa h$ is smaller for any case. In the limit $\kappa h=0$, the potential in the capillary is a constant equal to the surface potential of the wall for the specified case of $\bar{N}=1$. In the other limit with $\kappa h \rightarrow \infty$, the potential in the capillary becomes zero everywhere. For the case of $\bar{\zeta}=0$, the potential profile for fixed values of $b / h$ and $\kappa h$ increases monotonically with an increase in $\bar{N}$, which is not illustrated in Fig. 2, for conciseness. Comparing Fig. 2 with the analytical result for the potential profile obtained using the Debye-Hückel approximation [30], we find

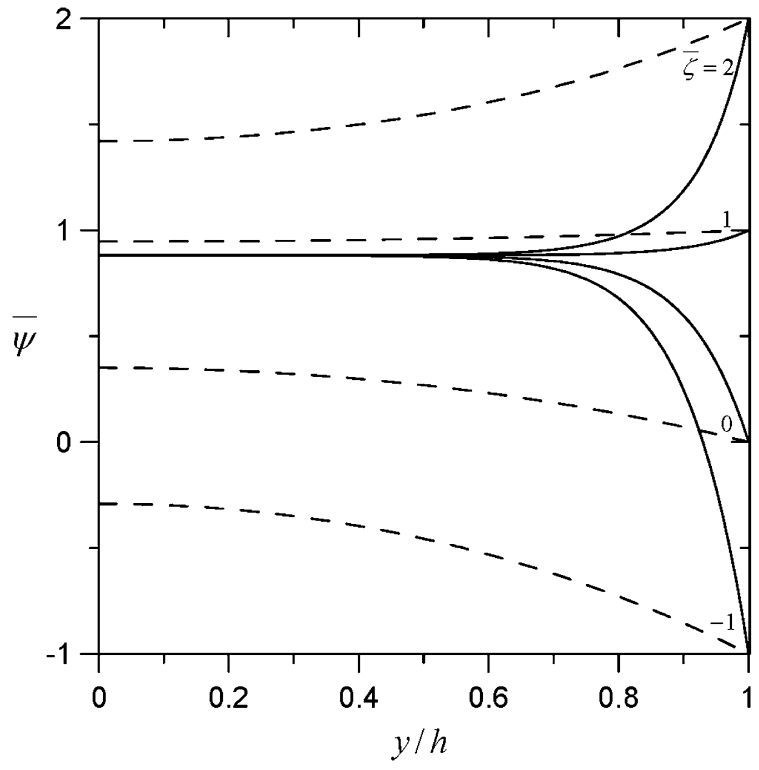

(a)

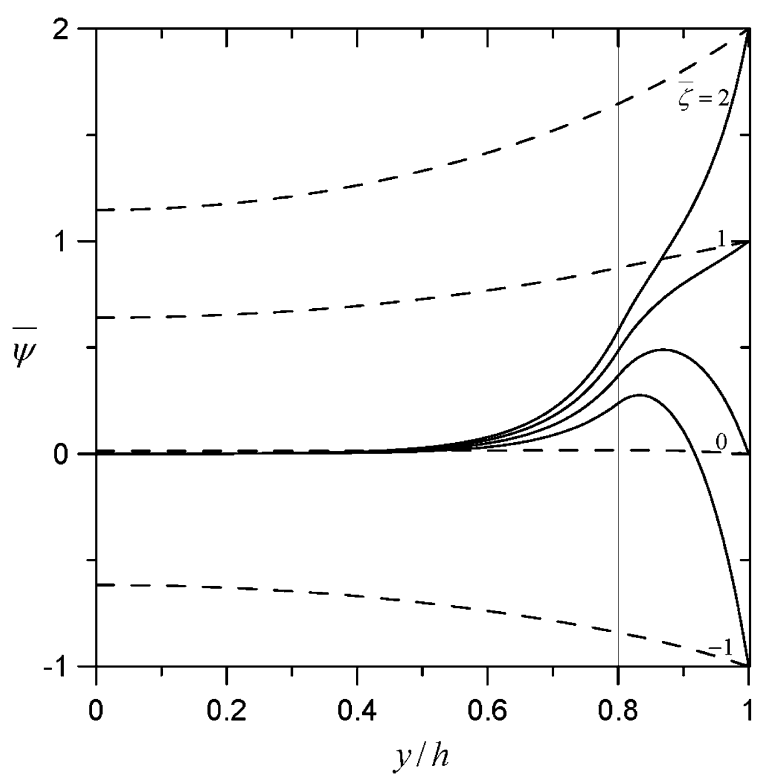

(b)

Fig. 2. Plots of the dimensionless potential $\bar{\psi}(y)$ in a slit with its inside walls coated with layers of polyelectrolytes with $\bar{N}=1$ versus the relative position $y / h$ : (a) $b / h=0$; (b) $b / h=0.8$. The solid and dashed curves denote the cases $\kappa h=10$ and $\kappa h=1$, respectively.

that this approximation in general overestimates the magnitudes of $\bar{\psi}$.

\section{Induced electric field distribution}

The ionic concentrations $n_{+}$and $n_{-}$in the fluid undergoing diffusioosmosis in the capillary slit with surface charge layers are not uniform in both axial $(z)$ and lateral $(y)$ directions; hence their prescribed gradients in the axial direction can give rise to a "diffusion current" distribution on a cross section of the slit. To prevent a continuous separation of the counterions and co-ions, an electric field distribution along the axial direction arises spontaneously in the electrolyte solution to produce 
another electric current distribution, which exactly balances the diffusion current [11-23]. This induced electric field generates an electroosmotic flow of the fluid in the slit, in addition to the chemiosmotic flow caused by the imposed electrolyte gradient directly. Both the chemiosmotic and the electroosmotic flows also generate an electric current distribution by ionic convection (known as the relaxation effect), and alternately, this secondary "convection current" again needs to be balanced by the electric current contributed from the induced electric field.

The total flux of either ionic species can be expressed in the general form

$\mathbf{J}_{ \pm}=-D_{ \pm}\left[\nabla n_{ \pm} \pm \frac{Z e}{k T} n_{ \pm}(\nabla \psi-\mathbf{E})\right]+n_{ \pm} \mathbf{u}$,

where $\mathbf{u}=u(y) \mathbf{e}_{z}$ is the fluid velocity in the axial direction of decreasing electrolyte concentration (i.e., $\mathbf{e}_{z}$ is the unit vector in the direction of $\left.-\nabla n^{\infty}\right), D_{+}$and $D_{-}$are the diffusion coefficients of the cations and anions, respectively, $\mathbf{E}=E(y) \mathbf{e}_{z}$ is the macroscopic electric field induced by the prescribed concentration gradient of the electrolyte, and the principle of superposition for the electric potential is used. To have no net electric current arising from the cocurrent diffusion, electric migration, and diffusioosmotic convection of the cations and anions, one must require that $\mathbf{J}_{+}=\mathbf{J}_{-}=\mathbf{J}$ (obviously, the lateral component of $\mathbf{J}$ vanishes and the ionic fluxes induced by $\nabla \psi$ in Eq. (5) are balanced by the lateral components of the diffusive ionic fluxes as required by the Boltzmann distribution given by Eq. (2)).

Applying the constraint $\mathbf{J}_{+}=\mathbf{J}_{-}$to Eq. (5), we obtain

$$
\begin{aligned}
\mathbf{E}= & \frac{k T}{Z e} \frac{\nabla n^{\infty}}{n^{\infty}(0)}\left[\frac{(1+\beta) e^{-\bar{\psi}}-(1-\beta) e^{\bar{\psi}}}{(1+\beta) e^{-\bar{\psi}}+(1-\beta) e^{\bar{\psi}}}\right. \\
& \left.+\frac{P e \sinh \bar{\psi}}{(1+\beta) e^{-\bar{\psi}}+(1-\beta) e^{\bar{\psi}}} \frac{u}{U^{*}}\right],
\end{aligned}
$$

where

$U^{*}=\frac{\varepsilon\left|\nabla n^{\infty}\right|}{\eta n^{\infty}(0)}\left(\frac{k T}{Z e}\right)^{2}=\frac{2 k T}{\eta \kappa^{2}}\left|\nabla n^{\infty}\right|$,

which is a characteristic value of the diffusioosmotic velocity,

$\beta=\frac{D_{+}-D_{-}}{D_{+}+D_{-}}$,

$\mathrm{Pe}=\frac{4 n^{\infty}(0) U^{*}}{\left(D_{+}+D_{-}\right)\left|\nabla n^{\infty}\right|}=\frac{8 n^{\infty}(0) k T}{\left(D_{+}+D_{-}\right) \eta \kappa^{2}}$,

and $\eta$ is the fluid viscosity, which is taken to be constant. As it is defined by Eq. (8), $-1 \leqslant \beta \leqslant 1$, with the upper and lower bounds occurring as $D_{-} / D_{+} \rightarrow 0$ and $\infty$, respectively. Typical values of the physical quantities in Eqs. (6)-(9) are $U^{*}=10^{-5} \mathrm{~m} / \mathrm{s}, D_{ \pm}=10^{-9} \mathrm{~m}^{2} / \mathrm{s}, n^{\infty}(0) /\left|\nabla n^{\infty}\right|=10^{-4} \mathrm{~m}$, and $\mathrm{Pe}$ of order unity. The induced electric field $\mathbf{E}$ given by Eq. (6) in a self-consistent way depends on the local electrostatic potential $\psi$, which was numerically solved in the previous section, and the fluid velocity $u$, which will be determined in the next section. It indicates that $\mathbf{E}$ is collinear with and proportional to the axially imposed electrolyte gradient $\nabla n^{\infty}$.
If we consider the situation that $\kappa b \gg 1$, then, at a position $y \ll b, \psi \rightarrow 0$ and Eq. (6) for the induced electric field caused by the imposed electrolyte concentration gradient reduces to its bulk-phase quantity,

$\mathbf{E}^{\infty}=\frac{k T}{Z e} \frac{\beta \nabla n^{\infty}}{n^{\infty}(0)}$.

For the special case of uncharged surface layers and slit walls $(\bar{N}=\bar{\zeta}=0), \mathbf{E}$ at any location $y$ is also identical to this bulkphase quantity. Note that $\mathbf{E}^{\infty}$ is in the same direction as $\beta \nabla n^{\infty}$ and linearly proportional to the parameter $\beta$, but $\mathbf{E}(y)$ need not vanish if $\beta=0$, even as $\mathrm{Pe}=0$, as shown in Eq. (6).

\section{Fluid velocity distribution}

We now consider the steady diffusioosmotic flow of a symmetric electrolyte solution in a capillary slit with each of its inside walls covered by a layer of charged polymers under the influence of a constant concentration gradient of the electrolyte prescribed axially. The momentum balances on the incompressible and Newtonian fluid in the $y$ and $z$ directions give

$\frac{\partial p}{\partial y}+Z e\left(n_{+}-n_{-}\right) \frac{\mathrm{d} \psi}{\mathrm{d} y}=0$,

$\eta \frac{\mathrm{d}^{2} u}{\mathrm{~d} y^{2}}-H(y) f u=\frac{\partial p}{\partial z}-Z e\left(n_{+}-n_{-}\right) E$,

where $p(y, z)$ is the dynamic pressure distribution and $f$ is the hydrodynamic friction coefficient in the polymer layer per unit volume of the fluid, which is assumed to be constant. Equation (12) is the Navier-Stokes/Brinkman equation, modified by adding a term for electrostatic force.

The boundary conditions for $u$ are

$\frac{\mathrm{d} u}{\mathrm{~d} y}(y=0)=0$,

$u\left(y=b^{-}\right)=u\left(y=b^{+}\right)$,

$\frac{\mathrm{d} u}{\mathrm{~d} y}\left(y=b^{-}\right)=\frac{\mathrm{d} u}{\mathrm{~d} y}\left(y=b^{+}\right)$,

$u(y=h)=0$.

Equations (13b) and (13c) express the continuity conditions of $u$ and of $\mathrm{d} u / \mathrm{d} y$ at the outer boundary of the surface charge layer [25-27]. In Eq. (13d), we have assumed that the shear plane coincides with the surface of the bare wall.

After the substitution of Eq. (2) into Eq. (11) based on the assumption that the equilibrium ionic distributions are not affected by the net electrolyte flux $\mathbf{J}$, which is warranted if $\left|\nabla n^{\infty}\right| / \kappa n^{\infty}(0) \ll 1$, the pressure distribution in a capillary slit with each of its inside walls coated with a layer of adsorbed polyelectrolytes can be determined as

$p=p_{0}+2 n^{\infty}(z) k T[\cosh \bar{\psi}-\cosh \bar{\psi}(0)]$.

Here, $p_{0}$ is the pressure at the median plane between the slit walls, which is a constant in the absence of applied pressure gradient, and the electric potential distribution $\bar{\psi}(y)$ has been solved numerically in Section 2. 


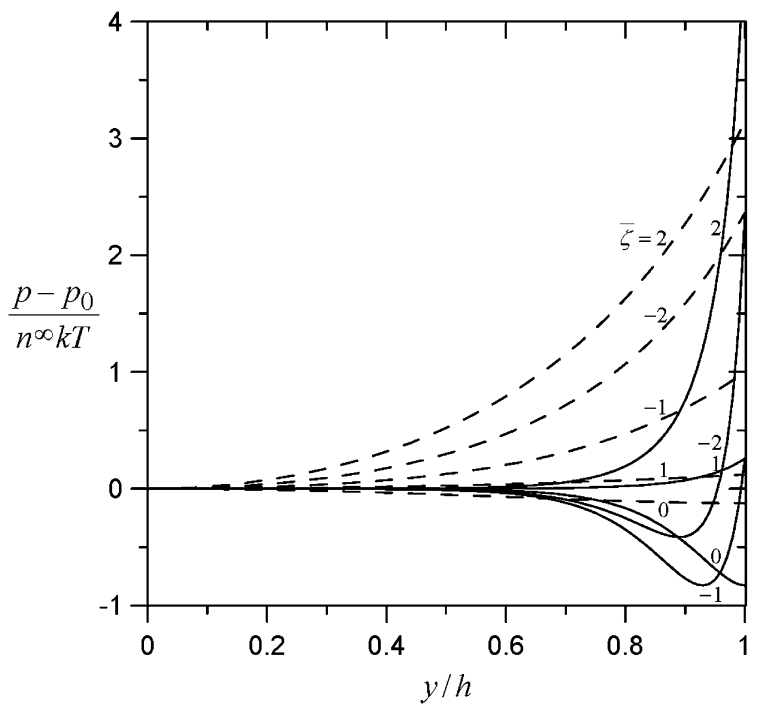

(a)

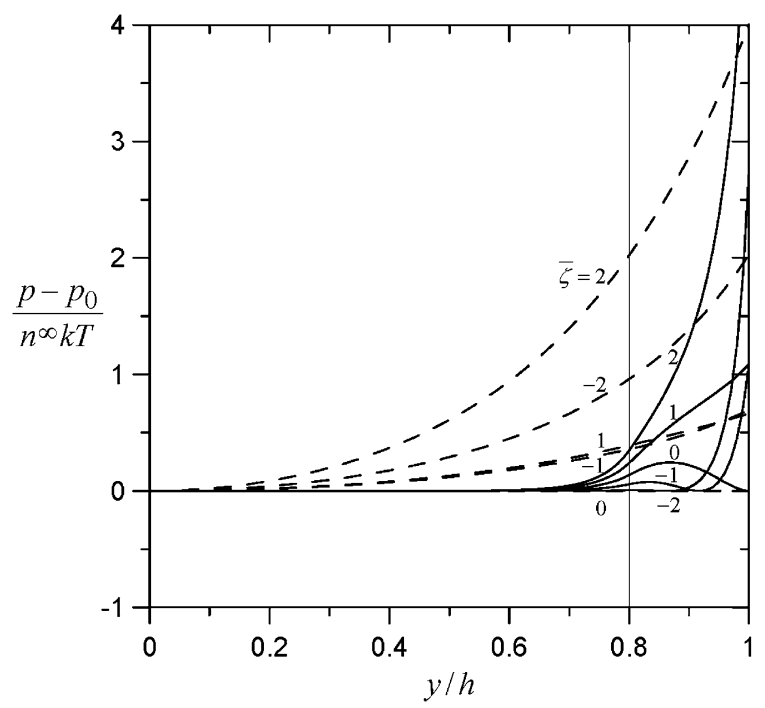

(b)

Fig. 3. Plots of the dimensionless pressure $\left(p-p_{0}\right) / n^{\infty} k T$ in a slit with its inside walls coated with layers of polyelectrolytes with $\bar{N}=1$ versus the relative position $y / h$ : (a) $b / h=0$; (b) $b / h=0.8$. The solid and dashed curves denote the cases $\kappa h=10$ and $\kappa h=1$, respectively.

The pressure distribution $p$ in the slit with surface charge layers can be obtained by substituting the numerical solution of $\bar{\psi}(y)$ into Eq. (14). Figs. 3a and 3b illustrate this result for the cases of $b / h$ equal to 0 and 0.8 , respectively, for $\bar{N}=1$ and various values of $\bar{\zeta}$ and $\kappa h$. It can be seen from Figs. 2 and 3 that the dimensionless pressure $\left(p-p_{0}\right) / n^{\infty} k T$ increases (decreases) with the relative coordinate $y / h$ as long as the magnitude of the dimensionless potential $\bar{\psi}$ increases (decreases) with $y / h$, irrespective of the values of $\bar{\zeta}$ and $\kappa h$. This behavior is understood by an examination of Eq. (11) for the fluid momentum balance in the lateral direction. For the case of $\zeta \geqslant 1$, the value of $\left(p-p_{0}\right) / n^{\infty} k T$ is always positive and increases monotonically with an increase in the value of $y / h$. On the other hand, for the case of $b / h=0$ and $\bar{\zeta}=0$, the value of $\left(p-p_{0}\right) / n^{\infty} k T$ is always negative, and its magnitude is also a monotonic increasing function of $y / h$. The value of $\left(p-p_{0}\right) / n^{\infty} k T$ may change signs when the value of $y / h$ increases and approaches unity for the case of $b / h=0$ and $\bar{\zeta}<0$. Comparing Fig. 3 with the analytical result for the pressure profile obtained using the Debye-Hückel approximation [30], we find that this approximation in general underestimates the values of $p-p_{0}$.

After substituting Eq. (2) for $n_{ \pm}$, Eq. (6) for $E(y)$, and Eq. (14) for $p$ into Eq. (12), and using the numerical solution for $\bar{\psi}(y)$ obtained in Section 2, the diffusioosmotic velocity distribution $u(y)$ of the electrolyte solution as a function of the parameters $b / h, \bar{N}, \bar{\zeta}, \beta, \mathrm{Pe}, \kappa h$, and $\lambda h$ [where $\lambda=$ $\left.(f / \eta)^{1 / 2}\right]$ can be numerically solved using the finite difference method. Then the distribution of the macroscopic electric field $E(y)$ induced by the prescribed electrolyte concentration gradient can also be numerically determined by substituting the numerical results for $\bar{\psi}(y)$ and $u(y)$ into Eq. (6). It is understood that, for given values of $b / h, \mathrm{Pe}, \kappa h, \lambda h$, and $y / h$, the quantities $E(y)$ and $u(y)$ with specified values $-\bar{\zeta}$ and $\beta$ are equal to those with the values $\bar{\zeta}$ and $-\beta$ when $\bar{N}=0$ (the polymer layer is uncharged), but these relations do not exist when $\bar{N} \neq 0$. Similarly, the quantities $E(y)$ and $u(y)$ with specified values $-\bar{N}$ and $\beta$ are equal to those with the values $\bar{N}$ and $-\beta$ when $\bar{\zeta}=0$. Note that the analytical results for $E(y)$ and $u(y)$ obtained using the Debye-Hückel approximation with no relaxation effect (taking $\mathrm{Pe}=0$ in Eq. (6)) are given in a previous article [31].

The parameter $1 / \lambda$ has the dimension of length and the square of it is the so-called Darcy permeability of the porous medium, which is related to the pore (or segment) size and porosity and characterizes the dynamic behavior of the viscous fluid in the porous medium. For the surface charge layers of human erythrocytes [26], rat lymphocytes [33], and grafted polymer microcapsules [34], experimental data of $1 / \lambda$ range from 1.35 to $3.7 \mathrm{~nm}$.

\section{Results and discussion}

The macroscopic electric field $E(y)$ induced by a prescribed concentration gradient of a symmetric electrolyte in a capillary slit filled with absorbed polyelectrolytes $(b / h=0)$ normalized by its quantity at the median plane between the slit walls, $E(0)$, as a function of the normalized coordinate $y / h$ is plotted in Figs. 4 and 5 for some values of the dimensionless parameters $\bar{N}, \bar{\zeta}, \beta, \kappa h, \lambda h$, and Pe. As expected, the normalized induced electric field in general is a sensitive function of $y / h$ and can deviate much from its bulk-phase value. This fact should play an important role in the electroosmotic contribution to the fluid velocity. When $\mathrm{Pe}=0$, the effect of the ionic convection on the induced electric field is not involved, as indicated in Eq. (6), and the induced electric field distribution is independent of the permeability parameter $\lambda h$. In this case, $E(y) / E(0)$ is positive and its value increases with an increase in $y / h$ from unity at the median plane of the slit to a maximum at its walls, increases with an increase in $\kappa h$ from unity at $\kappa h=0$ to a certain extent, decreases with an increase in $\bar{N}$ as $\beta=0$, and equals unity in the limits $\beta= \pm 1$, for an otherwise specified condition. On the other hand, when the value of Pe is finite, the magnitude 


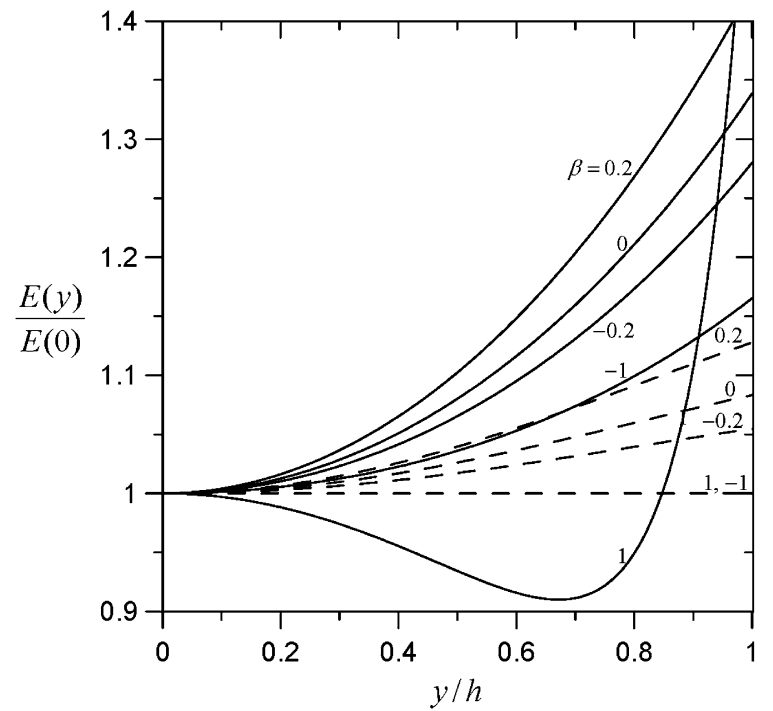

(a)

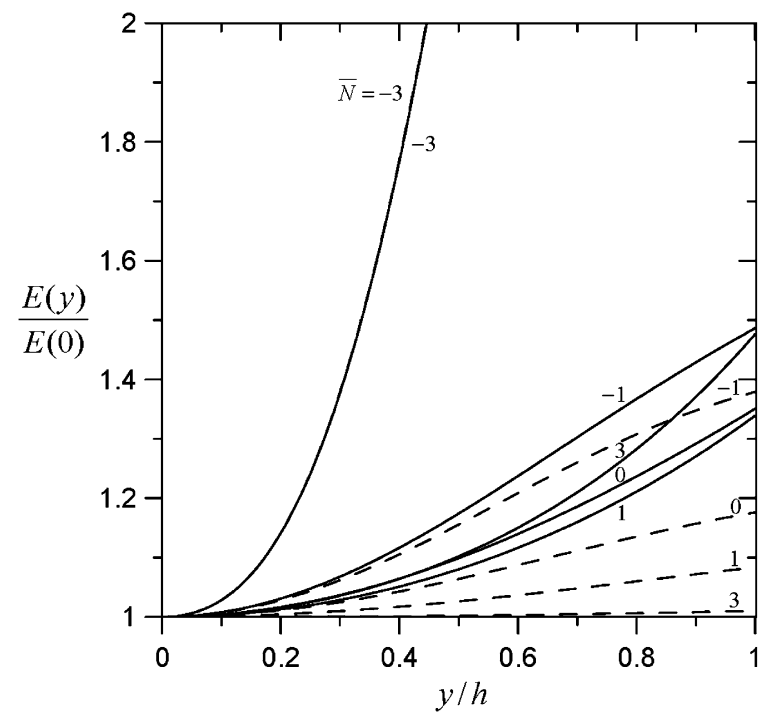

(b)

Fig. 4. Plots of the normalized electric field $E(y) / E(0)$ induced by an electrolyte gradient in a slit filled with polyelectrolytes $(b / h=0)$ with $\bar{\zeta}=2, \kappa h=1$, and $\lambda h=1$ versus the relative position $y / h$ : (a) for $\bar{N}=1$ and various values of $\beta$; (b) for $\beta=0$ and various values of $\bar{N}$. The solid and dashed curves represent the cases $\mathrm{Pe}=1$ and 0 , respectively.

of $E(y) / E(0)$ in general is larger than that for the case of Pe $=0$, but exceptions may exist and it may not be a monotonic function of $y / h$ as the magnitude of $\beta$ approaches unity, and it decreases with an increase in $\lambda h$ as expected. In all cases, the effect of the electrolyte convection (relaxation effect) on the local induced electric field in the capillary slit can be quite significant, not only quantitatively but also qualitatively, even for the cases of low fixed charge density in the layers of adsorbed polyelectrolytes and/or low zeta potential at the slit walls.

In Figs. 6 and 7, the normalized induced electric field $E(y) / E(0)$ in a slit with its inside walls covered by finite layers of adsorbed polyelectrolytes (with $b / h=0.8$ ) as a function of the relative coordinate $y / h$ is plotted for several values of the dimensionless parameters $\bar{N}, \bar{\zeta}, \beta, \kappa h, \lambda h$, and Pe. Interestingly, as indicated in Fig. $7 \mathrm{~b}, E(y) / E(0)$ decreases with an

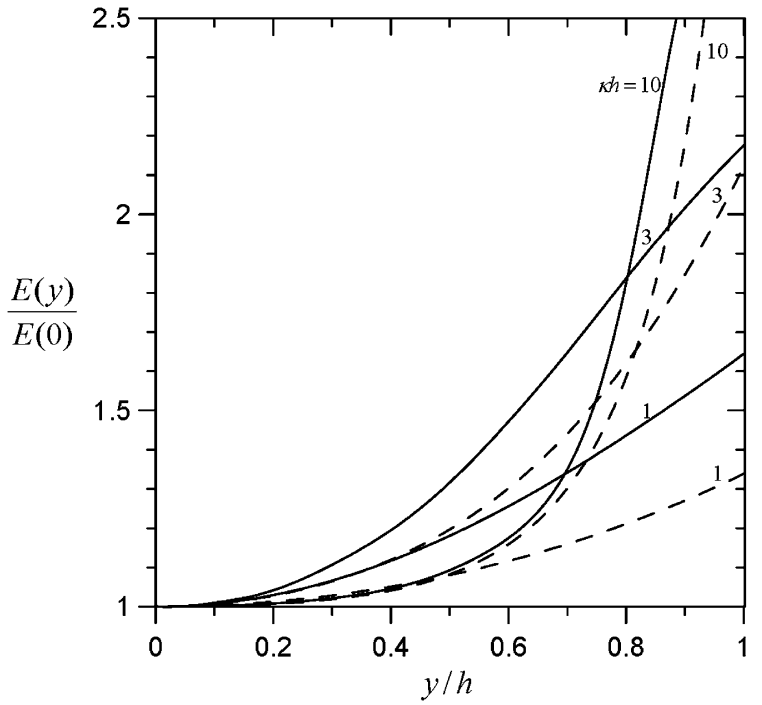

(a)

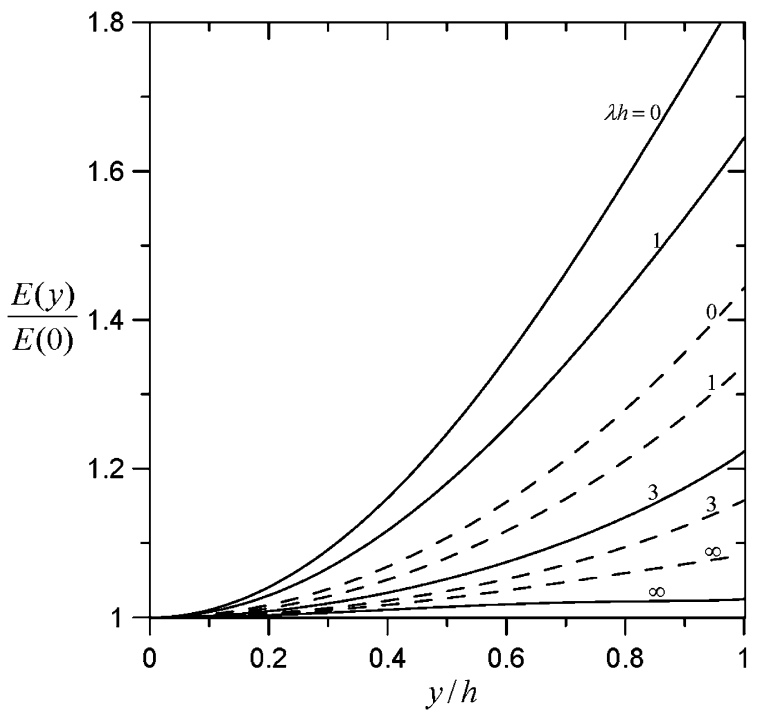

(b)

Fig. 5. Plots of the normalized electric field $E(y) / E(0)$ induced by an electrolyte gradient in a slit filled with polyelectrolytes $(b / h=0)$ with $\bar{N}=1$, $\beta=0$, and $\mathrm{Pe}=1$ versus the relative position $y / h$ : (a) for $\lambda h=1$ and various values of $\kappa h$; (b) for $\kappa h=1$ and various values of $\lambda h$. The solid and dashed curves represent the cases $\bar{\zeta}=6$ and 2 , respectively.

increase in the permeability parameter $\lambda h$ if the position $y$ is deep inside the porous surface layer, as expected, but it tends to increase with an increase in $\lambda h$ if the position $y$ is outside the porous layer. Since the thickness of the surface charge layer for the case considered in Fig. $6 \mathrm{~b}$ is relatively thin, its induced electric field distribution is not a sensitive function of the parameter $\bar{N}$, especially when the effect of electrolyte convection is included. Other characteristics of the induced electric field for the case with a finite value of $b / h$ are similar to those for the case of $b / h=0$. The existence of the surface charge layers at the slit walls can lead to an induced electric field distribution quite different from that in a slit with bare walls [17].

The dimensionless diffusioosmotic velocity $u / U^{*}$ of an electrolyte solution in a capillary slit filled with absorbed poly- 


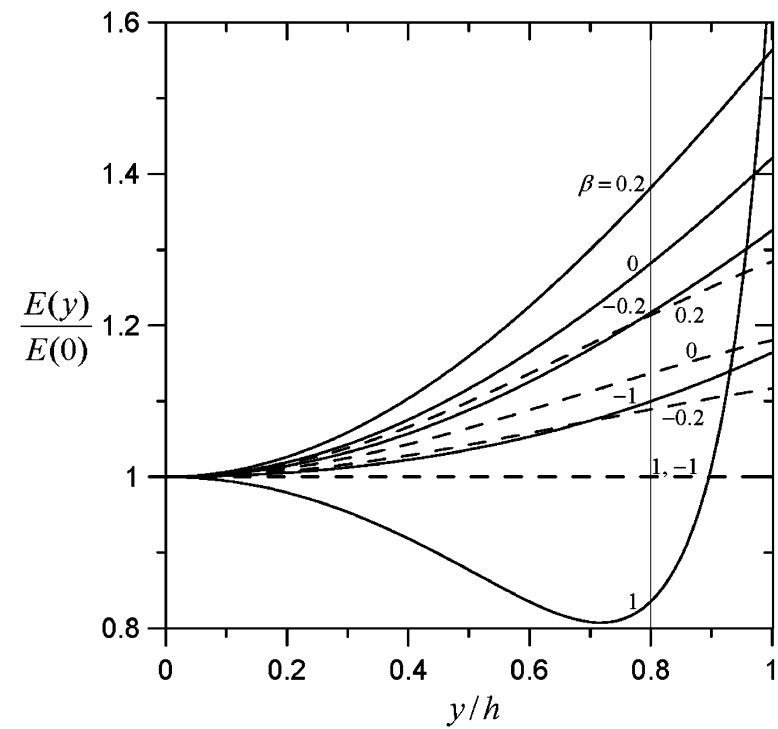

(a)

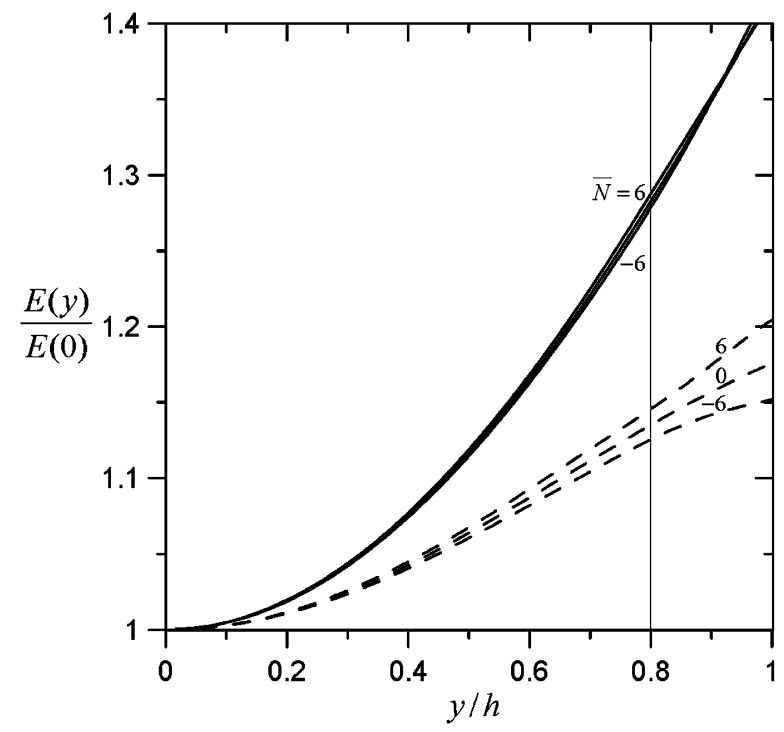

(b)

Fig. 6. Plots of the normalized electric field $E(y) / E(0)$ induced by an electrolyte gradient in a slit with surface layers of polyelectrolytes with $b / h=$ $0.8, \bar{\zeta}=2, \kappa h=1$, and $\lambda h=1$ versus the relative position $y / h$ : (a) for $\bar{N}=1$ and various values of $\beta$; (b) for $\beta=0$ and various values of $\bar{N}$. The solid and dashed curves denote the cases $\mathrm{Pe}=1$ and 0 , respectively.

electrolytes $(b / h=0)$ as a function of the relative coordinate $y / h$ is plotted in Figs. 8 and 9 for some values of the parameters $\bar{N}, \bar{\zeta}, \beta, \kappa h, \lambda h$, and Pe. This diffusioosmotic velocity can be either positive or negative and in general is a monotonic function of each parameter for an otherwise specified condition. For the typical case of $\bar{N}=1$ and $\bar{\zeta}=2$ given in Fig. 8a, $u$ is negative when the magnitude of $\beta$ is not too close to unity, meaning that the diffusioosmotic flow is in the direction of increasing electrolyte concentration. When the magnitude of $\beta$ approaches unity, the fluid may flow against the electrolyte concentration gradient ( $u$ is positive). In general, the magnitude of $u / U^{*}$ decreases with an increase in the normalized coordinate $y / h$ from the maximum at the median plane of the slit and to zero at the no slip walls (there are exceptions) for a given

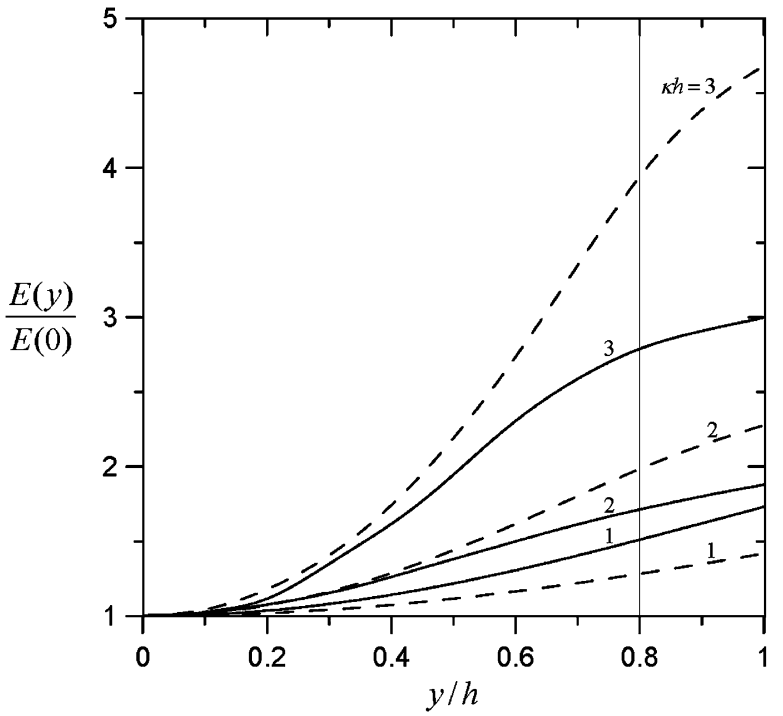

(a)

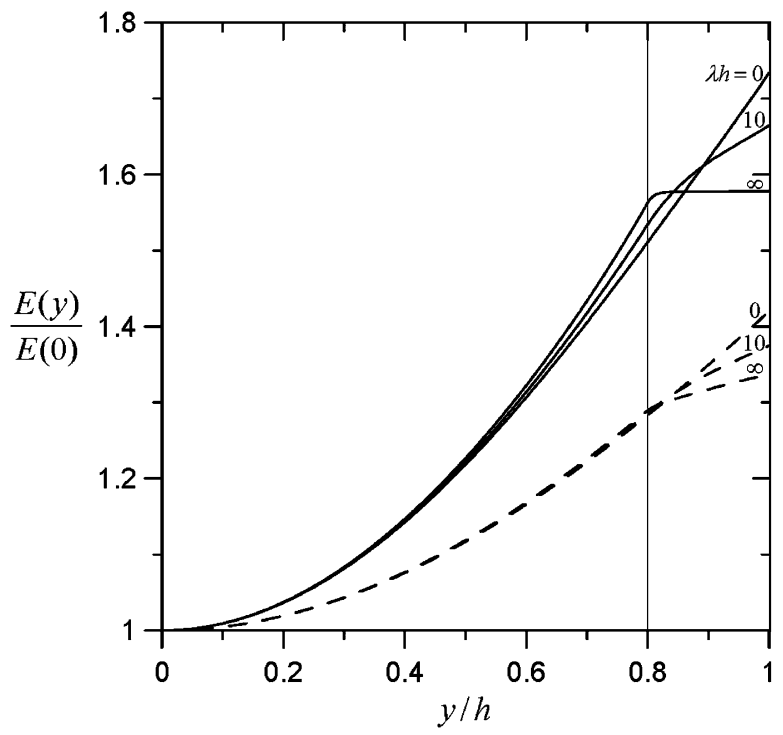

(b)

Fig. 7. Plots of the normalized electric field $E(y) / E(0)$ induced by an electrolyte gradient in a slit with surface layers of polyelectrolytes with $b / h=0.8$, $\bar{N}=1, \beta=0$, and $\mathrm{Pe}=1$ versus the relative position $y / h$ : (a) for $\lambda h=1$ and various values of $\kappa h$; (b) for $\kappa h=1$ and various values of $\lambda h$. The solid and dashed curves denote the cases $\bar{\zeta}=6$ and 2 , respectively.

system. As expected, Fig. 9b illustrates that the magnitude of the fluid velocity decreases as $\lambda h$ increases and vanishes as $\lambda h \rightarrow \infty$ because of the infinite friction of the polyelectrolytes. For all cases, the magnitude of $u(y) / U^{*}$ decreases with an increase in $\mathrm{Pe}$, and the effect of the ionic convection is significant and cannot be neglected.

In Figs. 10 and 11, the dimensionless diffusioosmotic velocity distribution $u(y) / U^{*}$ of an electrolyte solution in a slit with its inside walls covered by finite layers of adsorbed polyelectrolytes (with $b / h=0.8$ ) is plotted for several values of the parameters $\bar{N}, \bar{\zeta}, \beta, \kappa h, \lambda h$, and Pe. Similarly to the induced electric field distribution shown in Fig. 6b, Fig. 10b illustrates that the fluid velocity profile in the slit with relatively thin surface charge layers is not a sensitive function of the para- 


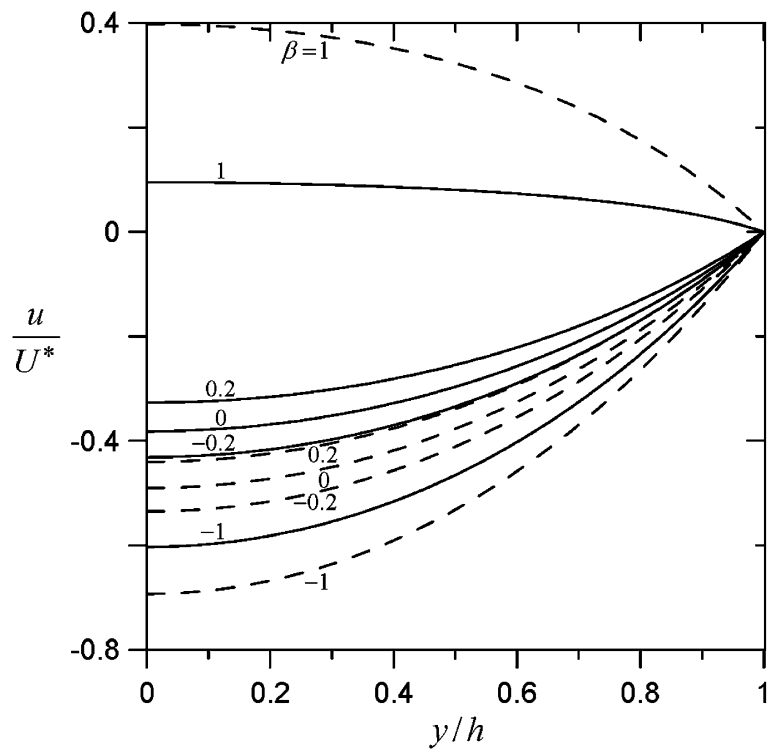

(a)

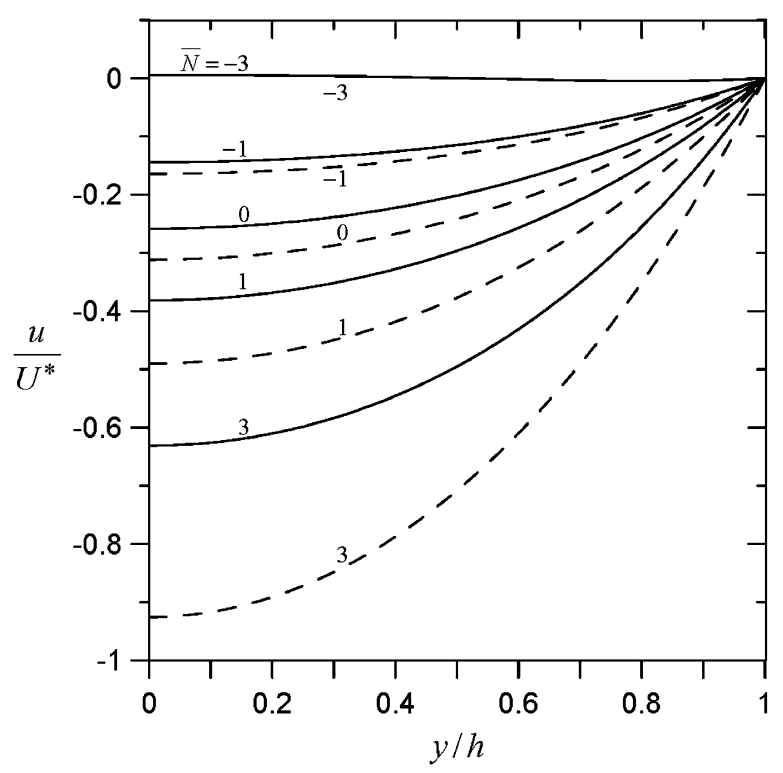

(b)

Fig. 8. Plots of the dimensionless diffusioosmotic velocity $u(y) / U^{*}$ in a slit filled with polyelectrolytes $(b / h=0)$ with $\bar{\zeta}=2, \kappa h=1$, and $\lambda h=1$ versus the relative position $y / h$ : (a) for $\bar{N}=1$ and various values of $\beta$; (b) for $\beta=0$ and various values of $\bar{N}$. The solid and dashed curves represent the cases $\mathrm{Pe}=1$ and 0 , respectively.

meter $\bar{N}$. For some cases, such as indicated by the curves with $\kappa h \geqslant 3$ in Fig. 11a, the diffusioosmotic velocity at the positions deep inside the porous surface layer can reverse direction from that at the positions outside the porous layer. In the limit $\lambda h \rightarrow \infty, u(y) / U^{*}$ vanishes within the surface charge layers $(y>b)$ as expected, but can be finite at other locations in the slit, as shown in Fig. 11b. Other flow characteristics for the case with a finite value of $b / h$ are similar to those for the case of $b / h=0$. Again, all the results suggest that the relaxation effect on the diffusioosmotic velocity can be significant. The existence of the surface charge layers at the slit walls can lead to a quite different diffusioosmotic flow from that in a slit with bare walls [17].

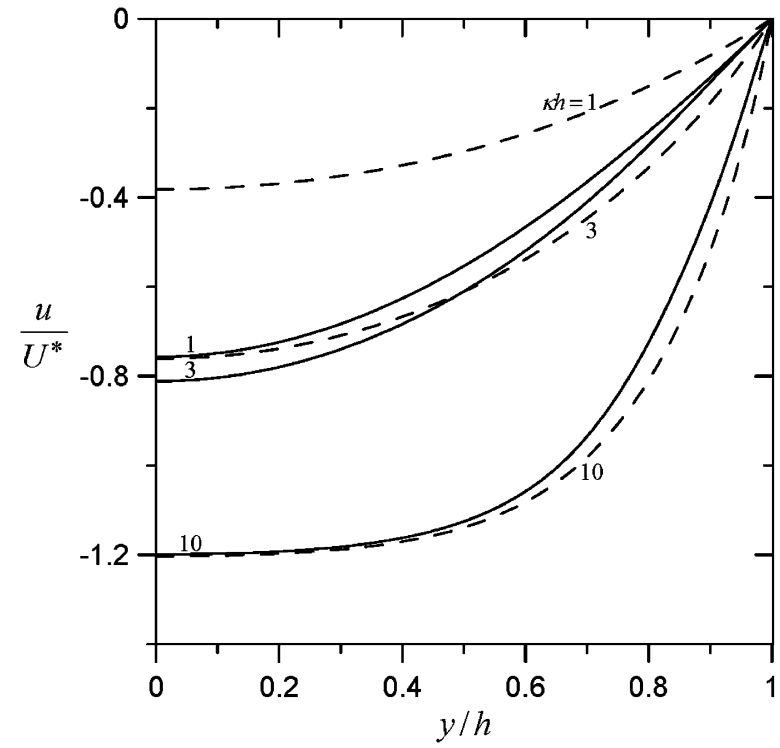

(a)

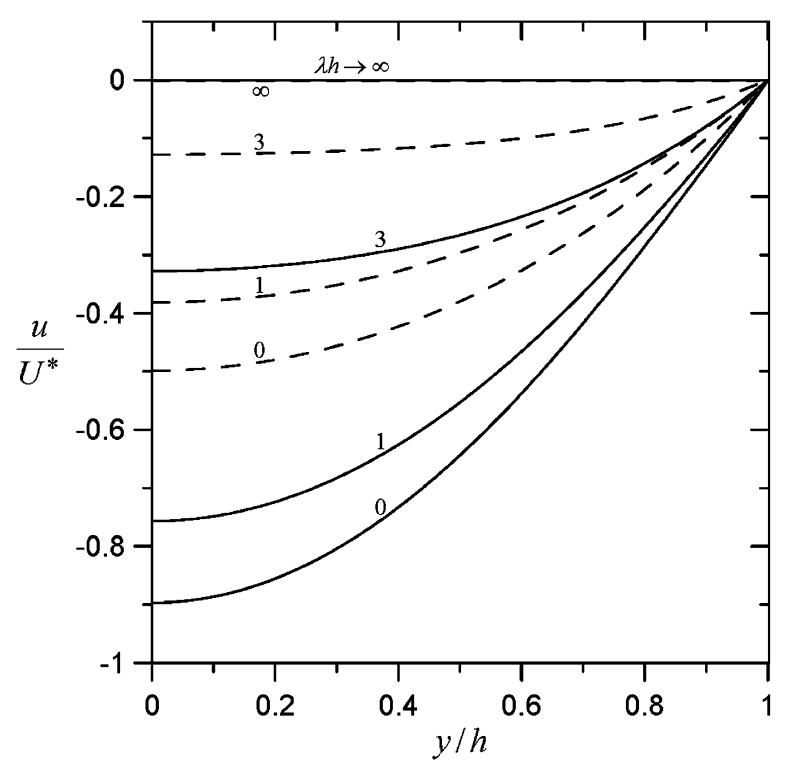

(b)

Fig. 9. Plots of the dimensionless diffusioosmotic velocity $u(y) / U^{*}$ in a slit filled with polyelectrolytes $(b / h=0)$ with $\bar{N}=1, \beta=0$, and $\mathrm{Pe}=1$ versus the relative position $y / h$ : (a) for $\lambda h=1$ and various values of $\kappa h$; (b) for $\kappa h=1$ and various values of $\lambda h$. The solid and dashed curves represent the cases $\bar{\zeta}=6$ and 2 , respectively.

In Fig. 12, the dimensionless diffusioosmotic velocity $u(0) / U^{*}$ of the electrolyte solution at the median plane of a capillary slit with its inside walls covered by finite layers of absorbed polyelectrolytes $(b / h=0.8)$ is plotted versus the parameter $\bar{N}$ at several values of $\beta, \lambda h$, and Pe for the case of $\bar{\zeta}=0$ and $\kappa h=10$. Maps showing the direction of this velocity for the case of $\bar{\zeta}=0, \kappa h=10$, and $\lambda h=1$ are also drawn in Fig. 13. The dependence of $u(0) / U^{*}$ on every parameter is quite similar to that of $u / U^{*}$ for a given value of $y / h$, and it is not necessarily a monotonic function of $\bar{N}$ for an otherwise specified condition. When the product of $\bar{N}$ and $\beta$ is negative (inside the second and fourth quadrants in Fig. 13), $u(0)$ is negative (as shown by example curves in the lower half of Fig. 12a 


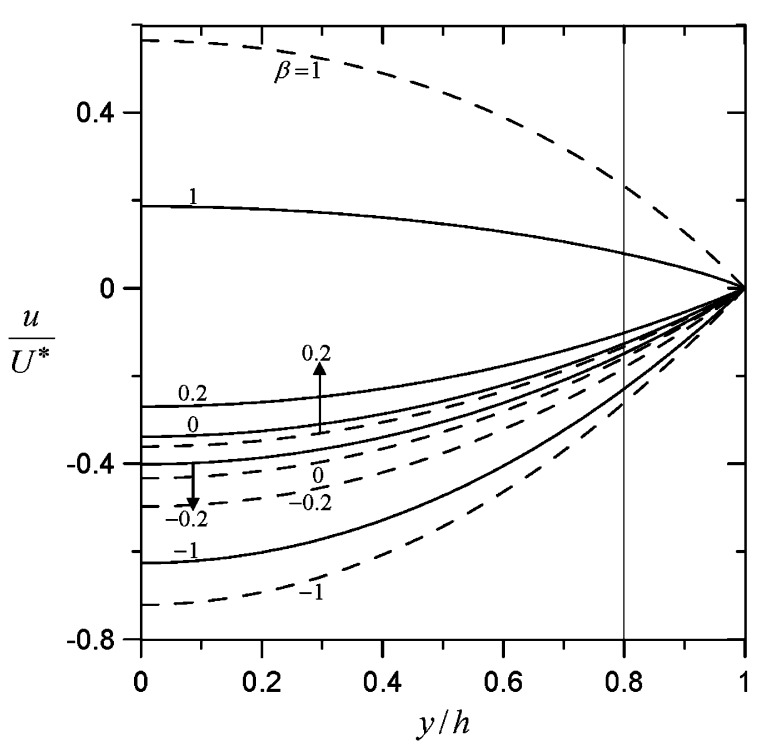

(a)

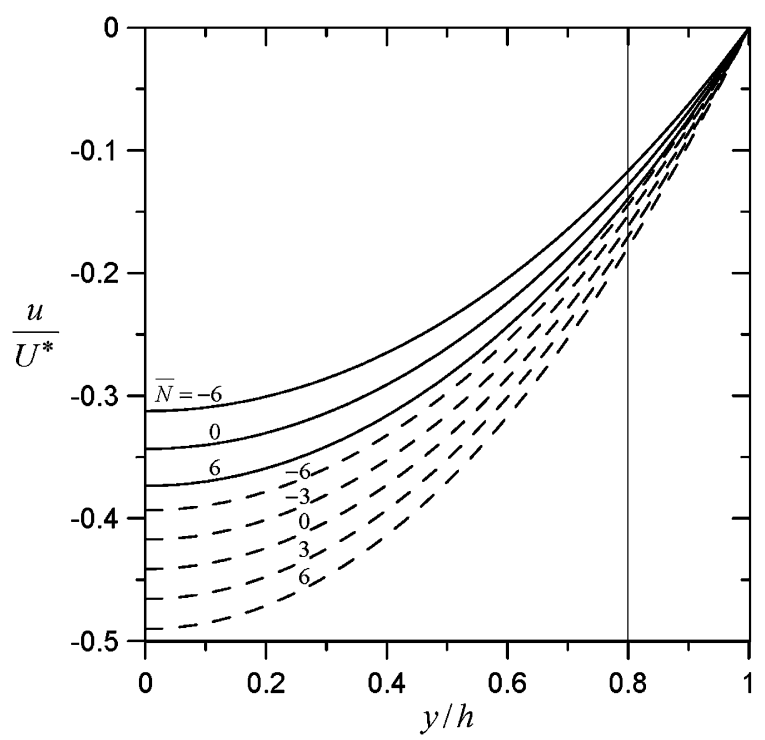

(b)

Fig. 10. Plots of the dimensionless diffusioosmotic velocity $u(y) / U^{*}$ in a slit with surface layers of polyelectrolytes with $b / h=0.8, \bar{\zeta}=2, \kappa h=1$, and $\lambda h=1$ versus the relative position $y / h$ : (a) for $\bar{N}=1$ and various values of $\beta$; (b) for $\beta=0$ and various values of $\bar{N}$. The solid and dashed curves denote the cases $P e=1$ and 0 , respectively.

or in the right half of Fig. 12b), and the electrolyte solution flows toward higher concentration. When the product of $\bar{N}$ and $\beta$ is positive (inside the first and third quadrants in Fig. 13), the diffusioosmotic velocity $u(0)$ may reverse its direction from along the concentration gradient to against it as $|\bar{\beta}|$ increases from zero to a certain extent for a given value of $\bar{N}$ (in addition to a reversal occurring at $\bar{N}=0$ ). When the value of Pe is finite, the probability of the diffusioosmotic flow of the electrolyte solution in the direction toward higher concentration for a combination of $\beta$ and $\bar{N}$ is reduced in comparison with the case of $\mathrm{Pe}=0$, due to the effect of the electrolyte convection. Again, Figs. 12 and 13 illustrate that this relaxation effect can be significant.

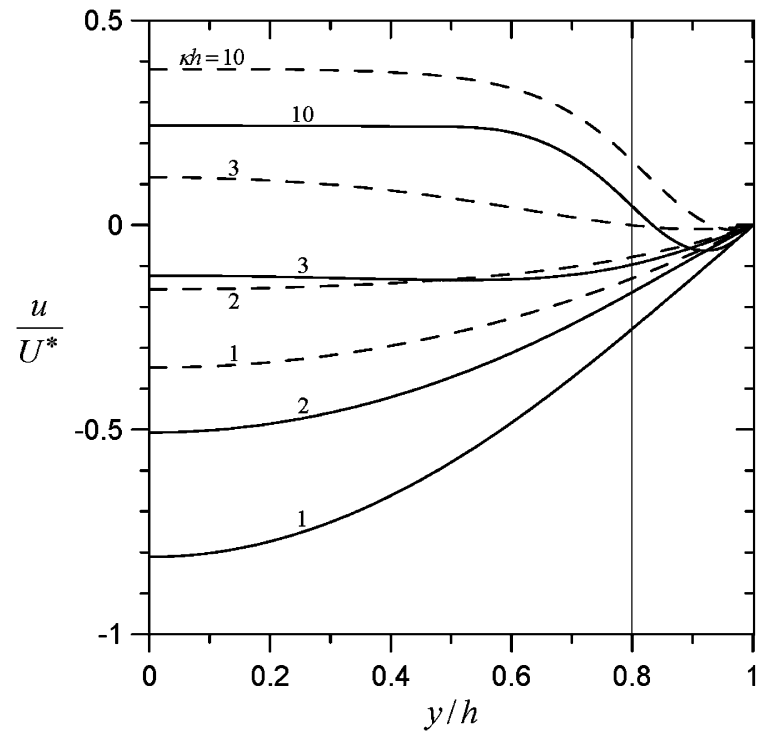

(a)

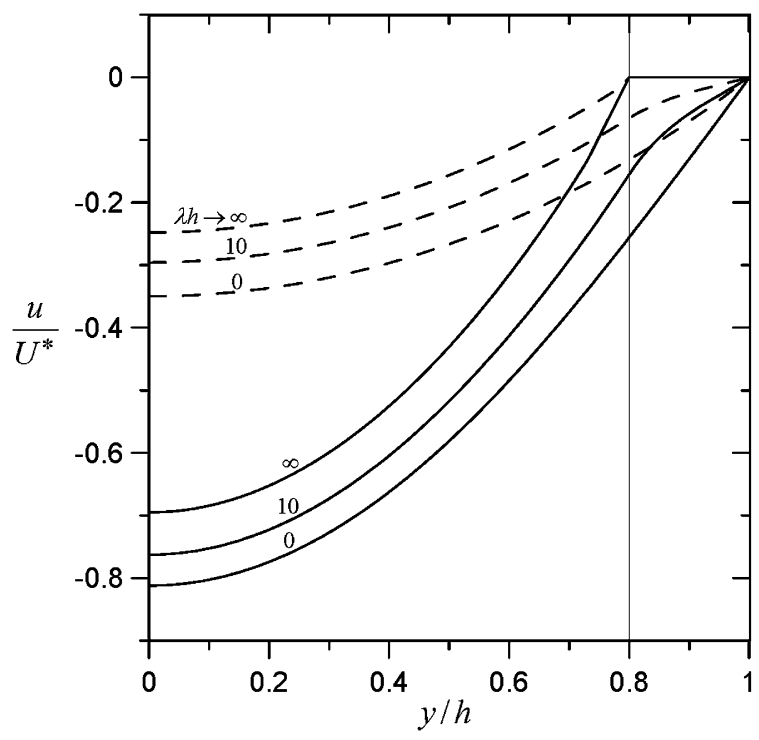

(b)

Fig. 11. Plots of the dimensionless diffusioosmotic velocity $u(y) / U^{*}$ in a slit with surface layers of polyelectrolytes with $b / h=0.8, \bar{N}=1, \beta=0$, and $\mathrm{Pe}=1$ versus the relative position $y / h$ : (a) for $\lambda h=1$ and various values of $\kappa h$; (b) for $\kappa h=1$ and various values of $\lambda h$. The solid and dashed curves denote the cases $\bar{\zeta}=6$ and 2, respectively.

\section{Concluding remarks}

A theoretical study of the steady diffusioosmotic flow of solutions of symmetric electrolytes in a capillary slit bearing a layer of adsorbed polyelectrolytes on each of its inside walls is presented in this work. No assumption is made about the thicknesses of the electric double layer and the surface charge layer. Both the effect of the lateral distribution of the electrolyte ions (or of the electrostatic potential) and the effect of ionic convection caused by the diffusioosmotic flow itself (relaxation effect) on the tangential electric field induced by the applied electrolyte concentration gradient are taken into account. By numerically solving the Poisson-Boltzmann equation and the modified Navier-Stokes/Brinkman equation applicable to the 


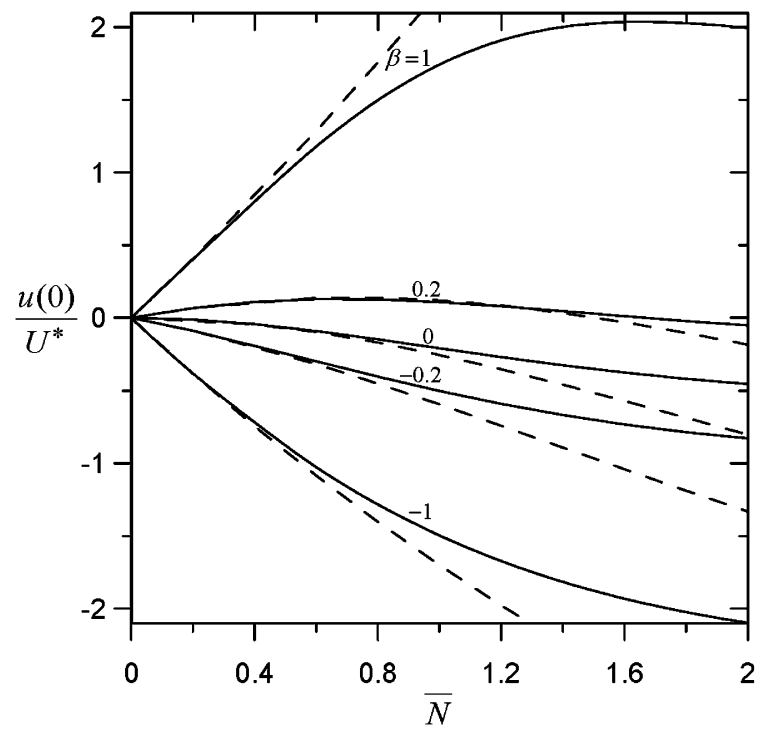

(a)

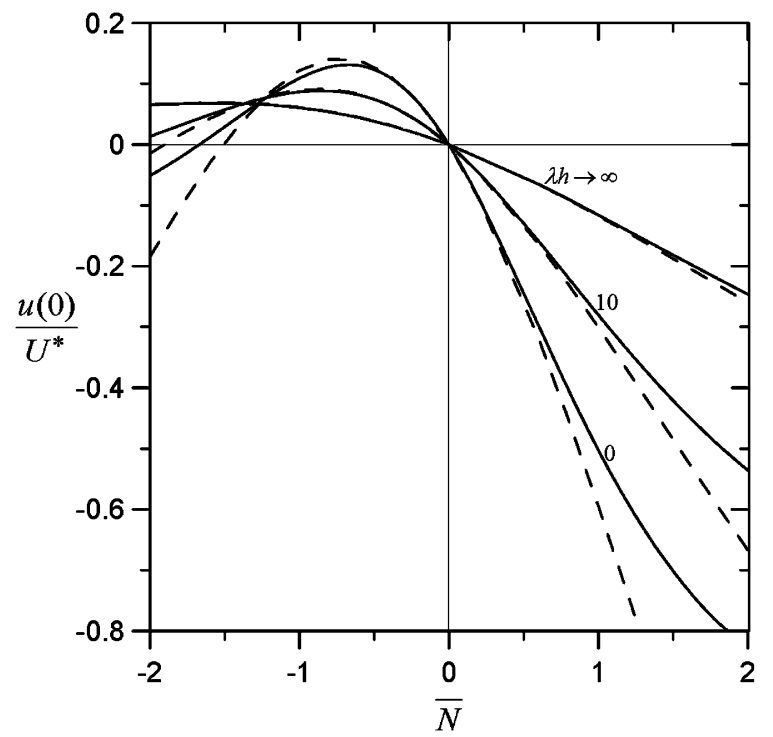

(b)

Fig. 12. Plots of the dimensionless diffusioosmotic velocity $u(0) / U^{*}$ at the median plane in a slit with surface layers of polyelectrolytes with $b / h=0.8$, $\bar{\zeta}=0$, and $\kappa h=10$ versus the parameter $\bar{N}$ : (a) for $\lambda h=1$ and various values of $\beta$; (b) for $\beta=-0.2$ and various values of $\lambda h$. The solid and dashed curves represent the cases $\mathrm{Pe}=1$ and 0 , respectively.

system, the electrostatic potential, dynamic pressure, induced electric field, and fluid velocity profiles on a cross section of the slit under the influence of a constant gradient of the electrolyte concentration are obtained. Our results demonstrate that the structure of the surface charge layer can lead to a diffusioosmotic flow quite different from that in a capillary with bare walls [17], depending on the characteristics of the capillary, of the surface charge layer, and of the electrolyte solution. The results show that the effect of the deviation of the local induced tangential electric field in the slit from its bulk-phase quantity and the relaxation effect due to electrolyte convection are very important and cannot be neglected in the evaluation of the diffusioosmotic velocity of electrolyte solutions in the axial direction of the capillary slit.

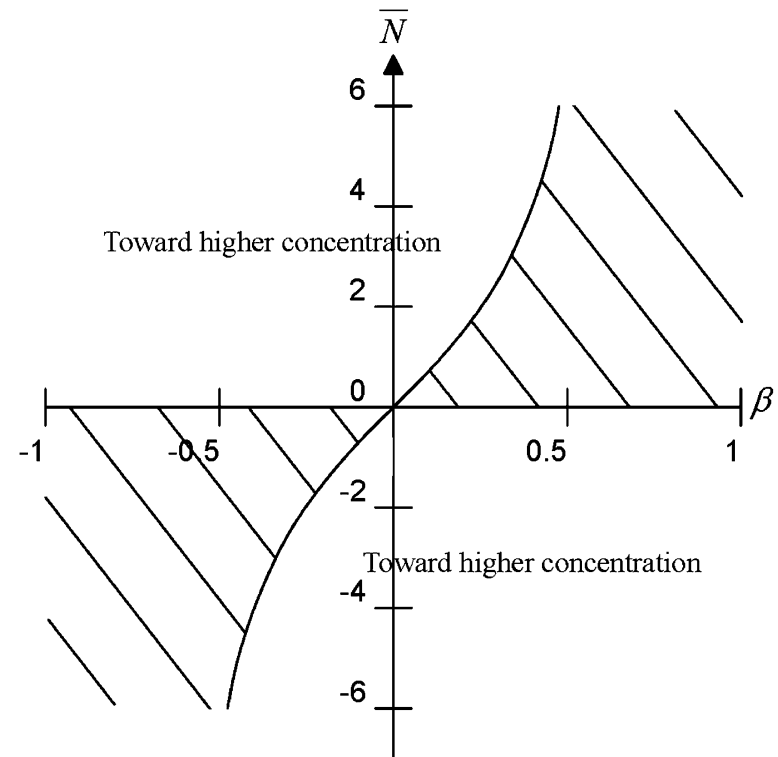

(a)

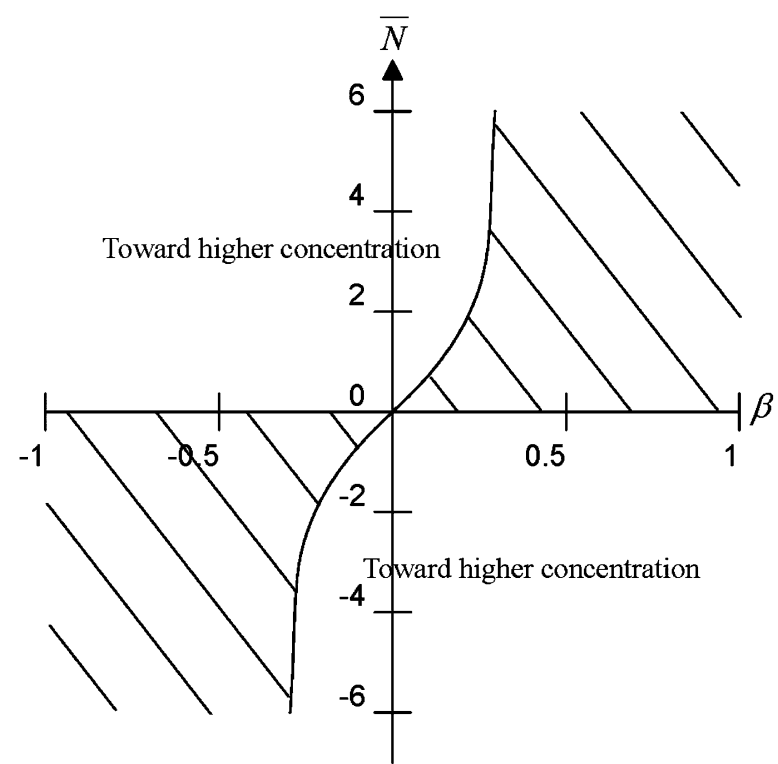

(b)

Fig. 13. Maps showing the direction of the diffusioosmotic velocity at the median plane in a slit with surface layers of polyelectrolytes with $b / h=0.8$, $\bar{\zeta}=0, \kappa h=10$, and $\lambda h=1$ : (a) $\mathrm{Pe}=0$; (b) $\mathrm{Pe}=1$.

It is worth repeating that all the results in this study are obtained on the basis of a small external gradient of the electrolyte concentration in the axial direction of the capillary slit. If the imposed concentration gradient $\left|\nabla n^{\infty}\right|$ is relatively large, then the effect of variation of the electrostatic potential $\psi$ in the slit with the tangential position cannot be neglected. However, it is reasonable to expect that this effect will lead to quantitatively rather than qualitatively different results.

\section{Acknowledgment}

This research was supported by the National Science Council of the Republic of China under Grant NSC95-2221-E-002281. 


\section{References}

[1] H. Helmholtz, Ann. Phys. (Leipzig) 7 (1879) 337.

[2] M. Smoluchowski, in: I. Graetz (Ed.), Handbuch der Electrizitat und des Magnetismus, vol. II, Barth, Leipzig, 1921, p. 336.

[3] D. Burgreen, F.R. Nakache, J. Phys. Chem. 68 (1964) 1084.

[4] C.L. Rice, R. Whitehead, J. Phys. Chem. 69 (1965) 4017.

[5] S.S. Dukhin, B.V. Derjaguin, in: E. Matijevic (Ed.), Surface and Colloid Science, vol. 7, Wiley, New York, 1974.

[6] C. Yang, D. Li, J. Colloid Interface Sci. 194 (1997) 95.

[7] D. Long, H.A. Stone, A. Ajdari, J. Colloid Interface Sci. 212 (1999) 338.

[8] A. Szymczyk, B. Aoubiza, P. Fievet, J. Pagetti, J. Colloid Interface Sci. 216 (1999) 285.

[9] H.J. Keh, H.C. Tseng, J. Colloid Interface Sci. 242 (2001) 450.

[10] Y.K. Wei, H.J. Keh, Colloids Surf. A 222 (2003) 301.

[11] B.V. Derjaguin, S.S. Dukhin, A.A. Korotkova, Kolloidn Zh. 23 (1961) 53.

[12] D.C. Prieve, J.L. Anderson, J.P. Ebel, M.E. Lowell, J. Fluid Mech. 148 (1984) 247.

[13] J.L. Anderson, Annu. Rev. Fluid Mech. 21 (1989) 61.

[14] Y. Pawar, Y.E. Solomentsev, J.L. Anderson, J. Colloid Interface Sci. 155 (1993) 488.

[15] H.J. Keh, S.B. Chen, Langmuir 9 (1993) 1142.

[16] H.J. Keh, H.C. Ma, Langmuir 21 (2005) 5461.

[17] H.C. Ma, H.J. Keh, J. Colloid Interface Sci. 298 (2006) 476.
[18] J.C. Fair, J.F. Osterle, J. Chem. Phys. 54 (1971) 3007.

[19] V. Sasidhar, E. Ruckenstein, J. Colloid Interface Sci. 85 (1982) 332.

[20] G.B. Westermann-Clark, J.L. Anderson, J. Electrochem. Soc. 130 (1983) 839.

[21] H.J. Keh, J.H. Wu, Langmuir 17 (2001) 4216.

[22] H.J. Keh, H.C. Ma, Colloids Surf. A 233 (2004) 87.

[23] H.J. Keh, H.C. Ma, Langmuir 23 (2007) 2879.

[24] S.S. Dukhin, Adv. Colloid Interface Sci. 44 (1993) 1.

[25] E. Donath, A. Voigt, J. Colloid Interface Sci. 109 (1986) 122.

[26] H. Ohshima, T. Kondo, J. Colloid Interface Sci. 135 (1990) 443

[27] V.M. Starov, Y.E. Solomentsev, J. Colloid Interface Sci. 158 (1993) 166.

[28] H.J. Keh, Y.C. Liu, J. Colloid Interface Sci. 172 (1995) 222.

[29] H.J. Keh, J.M. Ding, J. Colloid Interface Sci. 263 (2003) 645.

[30] J.H. Wu, H.J. Keh, Colloids Surf. A 212 (2003) 27.

[31] H.C. Ma, H.J. Keh, Colloids Surf. A 267 (2005) 4.

[32] K.A. Sharp, D.E. Brooks, Biophys. J. 47 (1985) 563.

[33] K. Morita, N. Muramatsu, H. Ohshima, T. Kondo, J. Colloid Interface Sci. 147 (1991) 457.

[34] O. Aoyanagi, N. Muramatsu, H. Ohshima, T. Kondo, J. Colloid Interface Sci. 162 (1994) 222

[35] A.W. Adamson, Physical Chemistry of Surfaces, fifth ed., Wiley, New York, 1990.

[36] S.S. Dukhin, R. Zimmermann, C. Werner, J. Colloid Interface Sci. 274 (2004) 309. 